\title{
Glauber and Kawasaki Dynamics for Determinantal Point Processes in Discrete Spaces
}

\author{
Myeongju CHAE and Hyun Jae YOO* \\ Department of Applied Mathematics, Hankyong National University, \\ 67 Seokjeong-dong, Anseong-si, Gyeonggi-do 456-749, Korea
}

Received February 27, 2009; final version accepted June 4, 2009

\begin{abstract}
We construct the equilibrium Glauber and Kawasaki dynamics on discrete spaces which leave invariant certain determinantal point processes. We will construct Fellerian Markov processes with specified core for the generators. Further, we discuss the ergodicity of the processes.
\end{abstract}

KEYWORDS: Glauber dynamics, Kawasaki dynamics, determinantal point process, Papangelou intensity, invariant measure, ergodic process

\section{Introduction}

In this paper we construct the equilibrium Glauber and Kawasaki dynamics on the discrete particle systems such that certain determinantal point processes are invariant under the Markov processes.

The determinantal point processes, or fermion point processes, are point processes whose correlation functions are given by determinants of kernel operators. It was invented by Macchi [13] and then has been extensively investigated by many people. It appears in many fields in mathematics and physics, for example, in random matrix theory and in fermion particle systems. For the details we refer to [10, 20,21,23] and references therein.

The construction of equilibrium dynamics is one of interesting subjects for the determinantal point processes. One approach is to construct the diffusion processes via Dirichlet forms [12]. During the last two decades there have been many works on the construction of equilibrium diffusion processes for Gibbs measures of interacting particle systems. We notice that the interacting Brownian particles of logarithmic potential, which is related to the determinantal point process of sine kernel and is called Dyson's model, was studied by Spohn [24]. Some related works to Dyson's model were also done recently in $[6,16]$. The diffusion process via Dirichlet form for the determinantal point processes in the non-percolating regime, or equivalently in the high temperature or small activity regime, was constructed by the second named author [25]. The other approach, in particular for particle systems, is to construct the particle birth and death processes, so called Glauber dynamics, and the particle jump processes, called Kawasaki dynamics. The general interacting particle systems in discrete model were developed in detail by Liggett [9]. The Glauber and Kawasaki dynamics for the continuum models have been investigated in the literature [3-5, 7, 8, 17]. Among these, Kondratiev et al. established the Dirichlet form approach for the construction of the equilibrium Glauber and Kawasaki dynamics for continuum systems so that the Gibbs measures for the system are invariant under the Markov processes [7, 8]. For instance, the standard superstable interaction of Ruelle [18] falls into their regime of applications. Lytvynov and Ohlerich applied the methods developed in $[7,8]$ to construct the equilibrium Glauber and Kawasaki dynamics that leave invariant certain determinantal point processes in continuum model [11]. The Glauber dynamics for discrete determinantal point processes was studied by Shirai and the second named author [22]. This paper can be regarded as a continuation of the work in [22]. We emphasize that the Markov processes constructed in this paper are Feller processes. That is, the semigroup acting on a continuous function gives rise to another continuous function. But the Markov processes constructed via Dirichlet forms are Hunt processes and the semigroups act on $L^{2}$-functions.

Let us briefly sketch the contents of this paper. We consider the infinite particle system, particles living on a discrete set, say $E$. We consider the determinantal point process $\mu$ on the configuration space $\mathcal{X}$. The point process $\mu$ has a defining kernel operator $K$ of the type $K:=A(I+A)^{-1}$, where $A$ is a positive definite, bounded linear operator on $l^{2}(E)$ that satisfies some hypothesis (H) (see Section 2). Under the hypothesis (H), $\mu$ is known to be Gibbsian [26]. The generators of Glauber and Kawasaki dynamics have the form (see subsection 2.1 for the details): 


$$
L^{(\mathrm{G})} f(\xi):=\sum_{x \in \xi} d(x ; \xi)[f(\xi \backslash x)-f(\xi)]+\sum_{y \in E \backslash \xi} b(y ; \xi)[f(y \xi)-f(\xi)]
$$

and

$$
L^{(\mathrm{K})} f(\xi):=\sum_{x \in \xi, y \in E \backslash \xi} c(x, y ; \xi)[f(y \xi \backslash x)-f(\xi)],
$$

where $d(x ; \xi)$ and $b(y ; \xi)$ are death and birth rates for Glauber dynamics, and $c(x, y ; \xi)$ are the jump rates for Kawasaki dynamics. In Subsection 3.1, following Liggett [9], we introduce the basic strategy to show the existence and ergodicity of the dynamics. Next for point processes with Papangelou intensities, we will give necessary conditions for the rate functions to satisfy the detailed balance condition (Theorem 3.3). Then we apply these ideas to our model. Given a DPP $\mu$ for a kernel operator which satisfies certain hypothesis, we give some concrete formulas for the rate functions (Proposition 3.5) and present the conditions for existence in the language of Papangelou intensities (Proposition 3.7). To guarantee that the conditions for the existence and ergodicity are satisfied, we need further stronger conditions introduced in Assumption (A) in Section 4. Under the assumption (A), we finally construct the dynamics of our purpose (Theorem 4.2). In the Appendix, we provide with a proof of a technical lemma, Lemma 4.1, which is worth to be noticed in itself.

Finally, comparing to [22], we would like to mention that in this paper the Kawasaki dynamics is included and the concept of Papangelou intensities is used in a crucial way to construct the dynamics. After denoting the detailed balance condition by Papangelou intensities, it is possible to choose the flip or jump rates for the equilibrium dynamics in many ways. In [22], we dealt with just one choice among them (see Remark 3.6). However, at the moment, we have to assume seemingly almost the same conditions as in [22]. Our future studies are addressed to

- the construction of the Glauber and Kawasaki dynamics under weaker conditions, at least under the hypothesis $(\mathrm{H})$;

- the investigation of the spectral gap, or log-Sobolev inequalities for the generators of the Markov processes.

\section{Preliminaries}

In this section we briefly recall the definition of Glauber and Kawasaki dynamics for spin systems, or equivalently lattice gases. Then we introduce the determinantal point process in discrete spaces and their Gibbsianness.

\subsection{Glauber and Kawasaki Dynamics for Lattice Gases}

Let $E$ be any countable set. We have in mind the system of the lattice space with $E=Z^{d}$, the $d$-dimensional integer lattice, but we deal with arbitrary discrete set $E$. Let $X$ be the set of all subsets $\xi \subset E$, called the configurations. For any subset $\Lambda \subset E$ and $\xi \in \mathcal{X}$, we denote by $\xi_{\Lambda}$ the restriction of $\xi$ to $\Lambda$ :

$$
\xi_{\Lambda}:=\xi \cap \Lambda \text {. }
$$

From now on if a subset $\Delta \subset E$ is finite, we denote it by $\Delta \subset \subset E$. For each $\Lambda \subset E$, let $\mathcal{F}_{\Lambda}$ be the $\sigma$-algebra on $\mathcal{X}$ generated by the functions $\xi \mapsto\left|\xi_{\Delta}\right|, \Delta \subset \subset \Lambda$, where $\left|\xi_{\Delta}\right|$ means the cardinality of the set $\xi_{\Delta}$. Thus we get an increasing system of $\sigma$-algebras $\left\{\mathcal{F}_{\Lambda}\right\}_{\Lambda \subset \subset E}$ and we let $\mathcal{F}:=\mathcal{F}_{E}$. Any probability measure $\mu$ on $(\mathcal{X}, \mathcal{F})$ is called a point process. We notice that the $\sigma$-algebra $\mathcal{F}$ on $\mathcal{X}$ can be understood as a Borel $\sigma$-algebra by a natural identification of $\mathcal{X}$ with $\{0,1\}^{E}$ equipped with the product topology. This identification is taken for granted in this paper, and consequently we will also consider $\mathcal{X}$ as a topological space. Notice in particular that $\mathcal{X}$ is a compact space.

Let $C(\mathcal{X})$ denote the set of all continuous functions on $\mathcal{X}$ equipped with the sup-norm $\|\cdot\|$. For $f \in C(\mathcal{X})$ and $x \in E$, let

$$
\Delta_{f}(x):=\sup \{|f(x \xi)-f(\xi)|: \xi \in \mathcal{X}\},
$$

where we have used a shorthand notation $x \xi:=\{x\} \cup \xi$. We define a subset of "smooth" functions $D(X)$ [9] by

$$
D(\mathcal{X}):=\left\{f \in C(\mathcal{X}):\||f|\|:=\sum_{x \in E} \Delta_{f}(x)<\infty\right\} .
$$

By the Stone-Weierstrass theorem, we easily see that $D(\mathcal{X})$ is dense in $C(\mathcal{X})$.

The generators for Glauber and Kawasaki dynamics are defined as follows. We first consider Glauber dynamics. For each $x \in E$, let $b(x ; \xi)$ and $d(x ; \xi)$ be nonnegative continuous functions on $\mathcal{X}$. They are called birth and death rates, respectively. Namely, given a configuration $\xi \in \mathcal{X}$, a particle is born at site $x \notin \xi$ in a rate $b(x ; \xi)$, and among the particles $\xi$ a particle at $x \in \xi$ dies out in a rate $d(x ; \xi)$. For each $x \in E$, we define

$$
c_{x}:=\sup _{\xi \ngtr x} \max \{b(x ; \xi), d(x ; x \xi)\},
$$

and define 


$$
c^{(\mathrm{G})}:=\sup _{x \in E} c_{x}
$$

Next for Kawasaki dynamics, we need the particle jump rates. For each $x \neq y \in E$, let $c(x, y$; $\xi)$ be a continuous function on $\mathcal{X}$ whose values are defined to be zero unless $x \in \xi$ and $y \notin \xi$. It is the rate for a particle at $x \in \xi$ to jump to the empty site $y \in E \backslash \xi$. We will also need to control the particle jump rates. For each pair $x \neq y \in E$, define

$$
\begin{aligned}
c_{\{x, y\}}:=\sup _{\xi \ngtr x, y} \max \{c(x, y ; x \xi), c(y, x ; y \xi)\} ; \\
c^{(\mathrm{K})}:=\sup _{x \in E} \sum_{y \neq x} c_{\{x, y\}} .
\end{aligned}
$$

We will assume that $c^{(\sharp)}<\infty$ for $\sharp=\mathrm{G}$ or $\mathrm{K}$. Under this assumption, the Markov pregenerators for Glauber and Kawasaki dynamics are defined as follows. For $f \in D(\mathcal{X})$, we define for Glauber dynamics

$$
L^{(\mathrm{G})} f(\xi):=\sum_{x \in \xi} d(x ; \xi)[f(\xi \backslash x)-f(\xi)]+\sum_{y \in E \backslash \xi} b(y ; \xi)[f(y \xi)-f(\xi)],
$$

and for Kawasaki dynamics

$$
L^{(\mathrm{K})} f(\xi):=\sum_{x \in \xi, y \in E \backslash \xi} c(x, y ; \xi)[f(y \xi \backslash x)-f(\xi)] .
$$

Here we have also used a short notation $\xi \backslash x$ for $\xi \backslash\{x\}$. For a definition and proof that $L^{(\sharp)}, \sharp=\mathrm{G}$ or $\mathrm{K}$, becomes a Markov pregenerator, we refer to [9, Definition 2.1 and Proposition 3.2].

In this paper we will investigate the conditions on the rates $b(x ; \xi), d(x ; \xi)$, and $c(x, y ; \xi)$ so that not only (the closure of) $L^{(\sharp)}, \sharp=\mathrm{G}$ or $\mathrm{K}$, becomes a Markov generator on $C(\mathcal{X})$ but also it leaves invariant certain point process $\mu$ on $(\mathcal{X}, \mathcal{F})$. We are concerned exclusively with determinantal point proccesses on $E$, which are briefly introduced in the next subsection.

\subsection{Determinantal Point Processes}

Determinantal point processes (DPP's hereafter), or fermion point processes, are the probability measures on the configuration space of particles. The particles can stay either on continuum spaces or on discrete sets. The correlation functions of DPP's are given by determinants of a priori given kernel operator as shown in Theorem 2.1 below. Typically, they have a fermionic nature, namely, the energy increases when new particles add into a given configuration of particles. For the basic theories of DPP's we refer to references [10, 13, 21, 23]. Here we follow the reference [21] for the introduction.

Let $E$ be the countable set in the previous subsection and let $K$ be a Hermitian positive definite, bounded linear operator on the Hilbert space $\mathscr{H}_{0}:=l^{2}(E)$, the set of square summable functions (sequences) on $E$ equipped with the usual inner product:

$$
(f, g)_{0}:=\sum_{x \in E} \overline{f(x)} g(x), \quad f, g \in \mathscr{H}_{0}
$$

The induced norm is denoted by $\|\cdot\|_{0}$. The following is an existence theorem for DPP's, which we present in the form stated in [21].

Theorem 2.1. Let $E$ be a countable set and $K$ a Hermitian bounded operator on $\mathscr{H}_{0}$. Assume that $0 \leq K \leq I$. Then, there exists a unique probability measure $\mu$ on $(\mathcal{X}, \mathcal{F})$ such that for any finite set $\left\{x_{1}, \ldots, x_{n}\right\} \subset E$, the $n$-th correlation function is given as follows:

$$
\rho_{\mu}^{(n)}\left(x_{1}, \ldots, x_{n}\right):=\mu\left(\left\{\xi \in \mathcal{X}: \xi \supset\left\{x_{1}, \ldots, x_{n}\right\}\right\}\right)=\operatorname{det}\left(K\left(x_{i}, x_{j}\right)\right)_{1 \leq i, j \leq n} .
$$

Next we discuss the density functions for DPP's. For each subset $\Lambda \subset E$, let $P_{\Lambda}$ denote the projection operator on $\mathscr{H}_{0}$ onto the subspace $l^{2}(\Lambda)$ and let $K_{\Lambda}:=P_{\Lambda} K P_{\Lambda}$ denote the restriction of $K$ onto the projection space. For each $\Lambda \subset \subset E$, assuming at the moment that $I_{\Lambda}-K_{\Lambda}$ is invertible, we define

$$
A_{[\Lambda]}:=K_{\Lambda}\left(I_{\Lambda}-K_{\Lambda}\right)^{-1} \text {. }
$$

The local marginals of the DPP $\mu$ corresponding to the operator $K$ are given by the formula: for each $\Lambda \subset \subset E$ and fixed $\xi \in \mathcal{X}$

$$
\mu_{\Lambda}\left(\xi_{\Lambda}\right):=\mu\left(\left\{\zeta: \zeta_{\Lambda}=\xi_{\Lambda}\right\}\right)=\operatorname{det}\left(I_{\Lambda}-K_{\Lambda}\right) \operatorname{det}\left(A_{[\Lambda]}(x, y)\right)_{x, y \in \xi_{\Lambda}},
$$

where $A_{[\Lambda]}(x, y), x, y \in \Lambda$, denotes the matrix components of $A_{[\Lambda]}$. We remark that the r.h.s. of (2.13) can be given a meaning even when $I_{\Lambda}-K_{\Lambda}$ is not invertible [21,23]. 


\subsection{Reproducing Kernel Hilbert Spaces, Papangelou Intensities and Gibbsianness of DPP's}

In this subsection we briefly discuss the Gibbsianness of DPP's. To show the Gibbsianness of a point process $\mu$ is equivalent to show the existence of global Papangelou intensities of $\mu[14,15,19,21]$. Since the Papangelou intensities are the key ingredients for the construction of equilibrium dynamics we review it for our model from [26]. We start by introducing a dual pair of reproducing kernel Hilbert spaces [1].

Let $A$ be a positive definite, bounded linear operator on $\mathscr{H}_{0} \equiv l^{2}(E)$ (the operator will define a DPP via the operator $\left.K:=A(I+A)^{-1}\right)$. We assume that $\operatorname{Ker} A=\{0\}$, so $\operatorname{Ran} A$ is dense in $\mathscr{H}_{0}$. We introduce two new inner products $(\cdot, \cdot)_{-}$ and $(\cdot, \cdot)_{+}$, respectively on $\mathscr{H}_{0}$ and $\operatorname{Ran} A$ as follows. First on $\mathscr{H}_{0}$, define

$$
(f, g)_{-}:=(f, A g)_{0}, \quad f, g \in \mathscr{H}_{0},
$$

and on $\operatorname{Ran} A$ define

$$
(f, g)_{+}:=\left(f, A^{-1} g\right)_{0}, \quad f, g \in \operatorname{Ran} A .
$$

The induced norms will be denoted by $\|\cdot\|_{-}$and $\|\cdot\|_{+}$, respectively. Let $\mathscr{H}_{-}$and $\mathscr{H}_{+}$be the completions of $\mathscr{H}_{0}$ and $\operatorname{Ran} A$ by the respective norms $\|\cdot\|_{-}$and $\|\cdot\|_{+}$. We then get the following rigging of Hilbert spaces:

$$
\mathscr{H}_{-} \supset \mathscr{H}_{0} \supset \mathscr{H}_{+} \text {. }
$$

We let $\mathrm{B}:=\left\{e_{x}\right\}_{x \in E}$ be the usual basis of $\mathscr{H}_{0}$, i.e., $e_{x}$ is a function on $E$ whose value at $x$ is 1 and the values at other sites are all zero. Let $A(x, y), x, y \in E$, be the representation of $A$ w.r.t. B. Then we notice that $\mathscr{H}_{+}$is a reproducing kernel Hilbert space (RKHS hereafter) with reproducing kernel (RK shortly) $A(x, y)$. That is, $\mathscr{H}_{+}$is a linear space of functions on $E$ with the following defining conditions:

(i) For every $x \in E$, the function $A(\cdot, x)$ belongs to $\mathscr{H}_{+}$;

(ii) The reproducing property: for every $x \in E$ and $g \in \mathscr{H}_{+}, g(x)=(A(\cdot, x), g)_{+}$.

We want $\mathscr{H}_{-}$to be also a RKHS (it is not the case in general), so we assume the hypothesis below throughout this paper:

Hypothesis $(\mathrm{H})$ : We suppose that $\mathscr{H}_{-}$is functionally completed [1], i.e., any vector of $\mathscr{H}_{-}$can be represented as a function on $E$.

For sufficient conditions for the hypothesis $(\mathrm{H})$ we refer to [26, Appendix]. Under the hypothesis $(\mathrm{H}), \mathscr{H}_{-}$becomes also a RKHS with RK, say $B(x, y), x, y \in E[1,26]$. Informally speaking, $B(x, y)$ is the matrix representation of $A^{-1}$, though it is not of bounded operator in general. In particular, under $(\mathrm{H})$ we have $e_{x} \in \mathscr{H}_{+}$for all $x \in E$ [26]. The flip or jump rates of our dynamics will be defined via the Papangelou intensities (defined below) of the DPP $\mu$ corresponding to the operator $K:=A(I+A)^{-1}$, which are in turn the squared norms of the vector-projections in the Hilbert space $\mathscr{H}_{-}$[26]. In particular, the interdependencies of rates, which must be controlled for the construction of the dynamics, are represented by the inner products in the restricted Hilbert spaces of $\mathscr{H}_{-}$ (see Proposition 3.8).

As mentioned above, we are concerned with DPP's corresponding to the operators $K:=A(I+A)^{-1}$. In order to get the Papangelou intensities, we need a variational principle [26]. For each $\Lambda \subset \subset E$, let

$$
\mathrm{F}_{\mathrm{loc}, \Lambda}:=\text { the linear space spanned by }\left\{e_{x}: x \in \Lambda\right\} \text {. }
$$

Let $x_{0} \in E$ be a fixed point and let $E=\left\{x_{0}\right\} \cup R_{1} \cup R_{2}$ be any partition of $E$ (one of $R_{1}$ and $R_{2}$ might be the empty set). For each $\Lambda \subset \subset E$, define

$$
\alpha_{\Lambda}:=\inf _{f \in \mathrm{F}_{\mathrm{loc}, \Lambda \cap R_{1}}}\left\|e_{x_{0}}-f\right\|_{-}^{2} \quad \text { and } \quad \beta_{\Lambda}:=\inf _{g \in \mathrm{F}_{\mathrm{loc}, \Lambda \cap R_{2}}}\left\|e_{x_{0}}-g\right\|_{+}^{2} .
$$

Obviously, $\left\{\alpha_{\Lambda}\right\}_{\Lambda \subset \subset E}$ and $\left\{\beta_{\Lambda}\right\}_{\Lambda \subset \subset E}$ are decreasing nets of nonnegative numbers. Consequently we define

$$
\alpha:=\lim _{\Lambda \uparrow E} \alpha_{\Lambda} \quad \text { and } \quad \beta:=\lim _{\Lambda \uparrow E} \beta_{\Lambda} .
$$

The variational principle says that, under the hypothesis $(\mathrm{H})$, no matter how we take a partition $E=\left\{x_{0}\right\} \cup R_{1} \cup R_{2}$, the product of $\alpha$ and $\beta$ is equal to 1 (see [26, Theorem 2.4] and also [21]):

$$
\alpha \beta=1 \text {. }
$$

The relation (2.20) guaranties, on the other hand, the existence of global Papangelou intensities. Let $\mu$ be the DPP corresponding to the operator $K:=A(I+A)^{-1}$, where $A$ satisfies the hypothesis $(\mathrm{H})$. Recall that the local Papangelou intensities of $\mu$ is defined as follows: for each $x \in E, x \in \Lambda \subset \subset E$, and $x \notin \xi \in \mathcal{X}$,

$$
\alpha_{[\Lambda]}\left(x ; \xi_{\Lambda}\right):=\frac{\mu_{\Lambda}\left(x \xi_{\Lambda}\right)}{\mu_{\Lambda}\left(\xi_{\Lambda}\right)} .
$$

The global Papangelou intensities are the limits 


$$
\alpha(x ; \xi):=\lim _{\Lambda \uparrow E} \alpha_{[\Lambda]}\left(x ; \xi_{\Lambda}\right),
$$

whenever the limit exists. The following theorem was proved in [26, Theorem 2.6]:

Theorem 2.2. Let the operator A satisfy the hypothesis $(H)$ and let $\mu$ be the DPP corresponding to the operator $K=A(I+A)^{-1}$. Then for all $x \in E$ and $x \notin \xi \in \mathcal{X}$, the Papangelou intensity $\alpha(x ; \xi)$ exists and it is equal to the number $\alpha$ in (2.19) obtained by replacing $x_{0}$ and $R_{1}$ in (2.18) by $x$ and $\xi$, respectively.

Remark 2.3. By (2.13) and (2.21)-(2.22) we see that

$$
\alpha(x ; \xi)=\lim _{\Lambda \uparrow E} \frac{\operatorname{det} A_{[\Lambda]}\left(x \xi_{\Lambda}, x \xi_{\Lambda}\right)}{\operatorname{det} A_{[\Lambda]}\left(\xi_{\Lambda}, \xi_{\Lambda}\right)},
$$

here $A_{[\Lambda]}\left(\xi_{\Lambda}, \xi_{\Lambda}\right)$ is the matrix $\left(A_{[\Lambda]}(x, y)\right)_{x, y \in \xi_{\Lambda}}$. What we have shown in the above theorem is that it is equal to the limit

$$
\alpha(x ; \xi)=\lim _{\Lambda \uparrow E} \frac{\operatorname{det} A\left(x \xi_{\Lambda}, x \xi_{\Lambda}\right)}{\operatorname{det} A\left(\xi_{\Lambda}, \xi_{\Lambda}\right)} .
$$

We will denote the dual relation (2.20) as

$$
\alpha(x ; \xi) \beta(x ; \xi)=1 .
$$

We notice also that for all $x \in E$ and $x \notin \xi \in \mathcal{X}$,

$$
\alpha(x ; \xi) \leq A(x, x) \leq\|A\| .
$$

\section{Construction of Glauber and Kawasaki Dynamics}

In this section we will construct the Glauber and Kawasaki dynamics for DPP's. We begin by briefly introducing the general strategy for the existence and ergodicity of the dynamics following [9].

\subsection{Existence and Ergodicity}

Throughout this subsection we assume that the flip rates $b(x ; \xi), d(x ; \xi)$, and jump rates $c(x, y ; \xi)$ are continuous functions for $\xi \in \mathcal{X}$ and satisfy the boundedness conditions $c^{(\#)}<\infty$, \# = G or K. Under these conditions the operators $L^{(\#)}, \#=\mathrm{G}$ or $\mathrm{K}$, in (2.8)-(2.9) are Markov pregenerators [9, Proposition 3.2, Chapter I].

The Markov pregenerators are closable [9, Proposition 2.5, Chapter I], but in order that the closures to be Markov generators, we need to control the interdependencies of the rate functions. For Glauber dynamics, following [9], we define for each $x \neq u \in E$,

$$
\gamma^{(\mathrm{G})}(x, u):=\sup _{\xi \ngtr x, u}\{|b(x ; \xi)-b(x ; u \xi)|+|d(x ; x u \xi)-d(x ; x \xi)|\} .
$$

And we define

$$
M^{(\mathrm{G})}:=\sup _{x \in E} \sum_{u \neq x} \gamma^{(\mathrm{G})}(x, u) .
$$

We will need also the lower bound of the flip rates defined by

$$
\varepsilon^{(\mathrm{G})}:=\inf _{x \in E} \inf _{\xi \ngtr x}\{b(x ; \xi)+d(x ; x \xi)\} .
$$

The interdependencies for Kawasaki dynamics will be given in the following way. First define for each $x \neq u \in E$

$$
\gamma^{(\mathrm{K})}(x, u):=\sum_{y \neq x} \sup _{\xi \ngtr x, y, u}\{|c(x, y ; x \xi)-c(x, y ; x u \xi)|,|c(y, x ; y \xi)-c(y, x ; y u \xi)|\} .
$$

Then we define

$$
M^{(\mathrm{K})}:=\sup _{x \in E} \sum_{u \neq x} \gamma^{(\mathrm{K})}(x, u)
$$

The lower bound of the rates for Kawasaki dynamics is given by

$$
\varepsilon^{(\mathrm{K})}:=\inf _{y \in E} \inf _{\xi \ngtr y}\left\{\sum_{x \in \xi} c(x, y ; \xi)+\sum_{x \notin y \xi} c(y, x ; y \xi)\right\} .
$$

Defining $\gamma^{(\#)}(x, x) \equiv 0$, we let $\Gamma^{(\#)}=\left(\gamma^{(\#)}(x, u)\right)_{x, u \in E}$ for \# $=\mathrm{G}$ or $\mathrm{K}$. The following theorem is proven by Liggett [9, Theorem 3.9, Chapter I]:

Theorem 3.1. For $\#=\mathrm{G}$ or $K$, assume that $c^{(\#)}<\infty$ and $M^{(\#)}<\infty$. Then we have

(a) The closure $\overline{L^{(\#)}}$ of $L^{(\#)}$ is a Markov generator of a Markov semigroup $T_{t}^{(\#)}$. 
(b) $D(X)$ is a core for $\overline{L^{(\#)}}$.

(c) For $f \in D(\mathcal{X})$

$$
\Delta_{T_{t}^{(\#)} f} \leq e^{-\varepsilon^{(\#)} t} \exp \left(t \Gamma^{(\#)}\right) \Delta_{f}
$$

(d) If $f \in D(\mathcal{X})$, then $T_{t}^{(\#)} f \in D(\mathcal{X})$ for all $t \geq 0$ and

$$
\left\|\left|T_{t}^{(\#)} f\right|\right\| \leq \exp \left[\left(M^{(\#)}-\varepsilon^{(\#)}\right) t\right]|| f \mid \| .
$$

Let us now introduce the concept of ergodicity. Recall that a point process $v$ on $(\mathcal{X}, \mathcal{F})$ is said to be invariant for the Markov process with semigroup $\left\{T_{t}, t \geq 0\right\}$ if

$$
\int T_{t} f d v=\int f d v
$$

for all $f \in C(\mathcal{X})$ and $t \geq 0$. A Markov process with semigroup $\left\{T_{t}, t \geq 0\right\}$ is said to be ergodic [9] if

(i) there is a unique invariant measure, say $v_{0}$;

(ii) $\lim _{t \rightarrow \infty} v T_{t}=v_{0}$ for all probability measure $v$ on $(\mathcal{X}, \mathcal{F})$.

Theorem 3.2 ([9, Theorem 4.1, Chapter I]). Suppose that the same conditions as in Theorem 3.1 hold. In addition, if $M^{(\#)}<\varepsilon^{(\#)}$ then the process is ergodic. Furthermore, for $g \in D(\mathcal{X})$,

$$
\left\|T_{t}^{(\#)} g-\int g d v_{0}^{(\#)}\right\| \leq c^{(\#)} \frac{e^{-\left(\varepsilon^{(\#)}-M^{(\#)}\right) t}}{\varepsilon^{(\#)}-M^{(\#)}}\|g\| \|,
$$

where $v_{0}^{(\#)}$ is the unique invariant measure.

\subsection{Detailed Balance Condition}

In this subsection we discuss the detailed balance conditions for Glauber and Kawasaki dynamics. Let $\mu$ be a point process and suppose that $\mu$ has Papangelou intensities $\alpha(x ; \xi)$. By the general theory, this implies that $\mu$ satisfies the DLR-conditions, which read as follows: for all bounded measurable functions $F: \mathcal{X} \rightarrow \mathbb{R}$ and $\Lambda \subset \subset E$,

$$
\int \mu(d \xi) F(\xi)=\int \mu(d \xi) \frac{1}{Z_{\Lambda}(\xi)} \sum_{\zeta_{\Lambda} \subset \Lambda} \alpha\left(\zeta_{\Lambda} ; \xi_{\Lambda^{c}}\right) F\left(\zeta_{\Lambda} \xi_{\Lambda^{c}}\right),
$$

where $Z_{\Lambda}(\xi)=\sum_{\zeta_{\Lambda} \subset \Lambda} \alpha\left(\zeta_{\Lambda} ; \xi_{\Lambda^{c}}\right)$ and $\alpha\left(\zeta_{\Lambda} ; \xi_{\Lambda^{c}}\right)=\prod_{i=1}^{\left|\zeta_{\Lambda}\right|} \alpha\left(x_{i} ; x_{1} \cdots x_{i-1} \xi_{\Lambda^{c}}\right)$. We refer to [14, 15] for more details.

The detailed balance condition for Glauber and Kawasaki dynamics w.r.t. $\mu$ means that the pregenerators $L^{(\sharp)}, \sharp=\mathrm{G}$ or $\mathrm{K}$, are symmetric:

$$
\int \mu(d \xi) f(\xi) L^{(\sharp)} g(\xi)=\int \mu(d \xi) L^{(\sharp)} f(\xi) g(\xi), \quad f, g \in D(X) .
$$

From the DLR-conditions (3.7) it is not hard to get equivalent conditions for the detailed balance. For simplicity, we assume that the Papangelou intensities $\alpha(x ; \xi)$ are positive.

Theorem 3.3. Let $L^{(\sharp)}, \sharp=G$ or $K$, be the Markovian pregenerators for Glauber and Kawasaki dynamics given in (2.8) and (2.9). In order that the detailed balance condition (3.8) is satisfied, it is necessary and sufficient that the rate functions satisfy

(a) for Glauber dynamics: for all $x \in E, x \notin \xi \in \mathcal{X}$,

$$
b(x ; \xi)=\alpha(x ; \xi) d(x ; x \xi)
$$

(b) for Kawasaki dynamics: $\forall x \neq y \in E$, and $\forall \xi \in \mathcal{X}$ with $x, y \neq \xi$,

$$
\alpha(x ; \xi) c(x, y ; x \xi)=\alpha(y ; \xi) c(y, x ; y \xi) .
$$

\subsection{Glauber and Kawasaki Dynamics for DPP's}

In this subsection, we will concretely define the flip and jump rates for Glauber and Kawasaki dynamics for DPP's, and then discuss the existence and ergodicity conditions for the dynamics.

From now on we fix an operator $A$ on $\mathscr{H}_{0}$ that satisfies the hypothesis (H). Thereby we also fix a DPP $\mu$ with kernel operator $K:=A(I+A)^{-1}$. We will construct Fellerian Markov generators for Glauber and Kawasaki dynamics on $C(\mathcal{X})$ that leave invariant the above DPP $\mu$. Recall the notations in subsection 2.1 . In order that the pregenerators (which are not yet explicitly defined) $L^{(\mathrm{G})}$ and $L^{(\mathrm{K})}$ in (2.8) and (2.9) would define Markovian generators for Feller processes, it is needed that the functions $b(x ; \xi), d(x ; \xi)$, and $c(x, y ; \xi)$ are continuous functions 
for $\xi \in \mathcal{X}$. These flip, or jump rates will be given via the Papangelou intensity function $\alpha(x ; \xi)$. So, we need the following

Lemma 3.4. For each $x \in E$, the Papangelou intensity $\alpha(x ; \xi)$ of $\mu$ is a continuous function of $\xi \in \mathcal{X}$.

Proof. Recall the definitions $\alpha_{\Lambda}$ and $\beta_{\Lambda}$ in (2.18). For each $x \in E$ and $\xi \in \mathcal{X}$ with $x \notin \xi$, we consider the partition $E=\{x\} \cup \xi \cup \bar{\xi}$, where $\bar{\xi}=E \backslash(x \xi)$. For each $\Lambda \subset \subset E$ we define local functions $\alpha_{\Lambda}\left(x ; \xi_{\Lambda}\right)$ and $\beta_{\Lambda}\left(x ; \xi_{\Lambda}\right)$ by the formula (2.18) replacing $x_{0}$ and $R_{1}$ by $x$ and $\xi$, respectively. As local functions, clearly the functions $\xi \mapsto \alpha_{\Lambda}\left(x ; \xi_{\Lambda}\right)$ and $\xi \mapsto \beta_{\Lambda}\left(x ; \xi_{\Lambda}\right)$ are continuous on the set $\{\xi \in \mathcal{X}: \xi \not \ngtr x\}$. Now as decreasing limits of continuous functions, the Papangelou intensity $\alpha(x ; \xi)$ and $\beta(x ; \xi)$ are both upper semi-continuous functions on $\{\xi \in \mathcal{X}: \xi \not \supset x\}$. Since $\alpha(x ; \xi)$ and $\beta(x ; \xi)$ are reciprocal to each other, they are also lower semi-continuous. This proves the lemma.

In the rest of the paper we will use the rate functions given in the following proposition, which we can easily prove. Recall the variational relation $\alpha(x ; \xi) \beta(x ; \xi)=1$ for all $x \in E$ and $x \notin \xi \in \mathcal{X}$ in (2.25).

Proposition 3.5. Assume the hypothesis $(H)$ and let $\mu$ be the DPP corresponding to the operator $K:=A(I+A)^{-1}$. Then the following choices for flip rates $b(x ; \xi)$ and $d(x ; \xi)$ for Glauber dynamics, and jump rates $c(x, y ; \xi)$ for Kawasaki dynamics are uniformly bounded for $\xi \in \mathcal{X}$ and satisfy the detailed balance conditions w.r.t. $\mu$ : (we let $x, y \notin \xi$ )

(a) for Glauber dynamics:

$$
b(x ; \xi):=\frac{\alpha(x ; \xi)}{1+\alpha(x ; \xi)} \quad \text { and } \quad d(x ; x \xi):=\frac{\beta(x ; \xi)}{1+\beta(x ; \xi)}
$$

(b) for Kawasaki dynamics:

$$
c(x, y ; x \xi)=d(x, y) \alpha(y ; \xi) g(\alpha(x ; \xi), \alpha(y ; \xi)),
$$

where $d(x, y)$ is a symmetric weight function, and $g: \mathbb{R}_{+}^{2} \rightarrow \mathbb{R}_{+}$is any symmetric, continuous function that makes $c(x, y ; x \xi)$ bounded.

The most simplest example for the function $g$ in the above is $g(u, v) \equiv 1$. However, reflecting on the nature of the dynamics, including this case, we may choose for any $0 \leq t \leq 1$,

$$
g(u, v) \equiv g_{t}(u, v):=\left(\frac{1}{(1+u)(1+v)}\right)^{t} .
$$

Then $c(x, y ; x \xi)$ becomes

$$
c(x, y ; x \xi)=d(x, y) \beta(x ; \xi)^{t} \alpha(y ; \xi)^{1-t}\left(\frac{1}{(1+\beta(x ; \xi))(1+\beta(y ; \xi))}\right)^{t} .
$$

Remark 3.6. In the equations (3.11) and (3.14), the terms $(1+\beta(x ; \xi))^{-1}$ and $((1+\beta(x ; \xi))(1+\beta(y ; \xi)))^{-t}$ are to make the flip rates bounded. When $\alpha(x ; \xi)$ is uniformly (for $\xi$ ) away from 0, we may drop these terms (taking $b(x ; \xi) \equiv \alpha(x ; \xi)$ and $d(x ; \xi) \equiv 1$ for Glauber dynamics). In [22], we have taken, in our terminology, $b(x ; \xi)=1+\alpha(x ; \xi)$ and $d(x ; x \xi)=1+\beta(x ; \xi)$ (see [22, eq. (1.9)]).

Now the flip rates for the equilibrium Glauber and Kawasaki dynamics have been given via the Papangelou intensities $\alpha(x ; \xi)$, we would like to represent the condition $M^{(\#)}<\infty$ in terms of $\alpha(x ; \xi)$. Let us assume that the weight function $d(x, y)$ in (3.12) satisfies

$$
0<d_{1}:=\inf _{x \in E} \sum_{y \neq x} d(x, y) \leq \sup _{x \in E} \sum_{y \neq x} d(x, y)=: d_{2}<\infty .
$$

Proposition 3.7. Suppose that the flip rates $b(x ; \xi)$ and $d(x ; \xi)$ and the jump rates $c(x, y ; x \xi)$ are given by the equations (3.11)-(3.12) with the function $g$ in (3.12) given by (3.13), and $d(x, y)$ satisfying (3.15). Define

$$
M_{1}^{(\mathrm{G})}:=\sup _{x \in E} \sum_{u \neq x} \sup _{\xi \ngtr x, u}(\alpha(x ; \xi)-\alpha(x ; u \xi))
$$

and

$$
M_{1}^{(\mathrm{K})}:=\sup _{x \in E} \sum_{u \neq x} \sum_{y \neq x} d(x, y) \sup _{\xi \ngtr x, y, u}[(\alpha(x ; \xi)-\alpha(x ; u \xi))+(\alpha(y ; \xi)-\alpha(y ; u \xi))] .
$$

Then $M^{(\#)} \leq a_{0} M_{1}^{(\#)}$ with a uniform constant $a_{0}$, and hence if $M_{1}^{(\#)}<\infty$ then $M^{(\#)}<\infty$ for \# $=\mathrm{G}$ or $K$, and all the results in Theorem 3.1 hold.

Proof. The proof immediately follows from the definitions. In particular, for the Kawasaki dynamics, we use the mean value theorem and the boundedness of the partial derivatives for the function $g_{t}(u, v)=((1+u)(1+v))^{-t}$. 
In order to get the boundedness of $M_{1}^{(\#)}$, \# = G or $\mathrm{K}$, we have to know the quantities $\alpha(x ; \xi)-\alpha(x ; u \xi)$ in (3.16)(3.17) more in detail. For that purpose we briefly introduce the restriction theory of reproducing kernel Hilbert spaces [1]. Let $\mathcal{H}$ be any RKHS (on $E$ ) with RK $C(x, y)$. Let $R \subset E$ be any (finite or infinite) subset of $E$, and let $C_{R}(x, y)$, $x, y \in R$, denote the restriction of $C$ to the set $R$. It was shown by Aronszajn that $C_{R}(x, y)$ is the RK of the RKHS, call it $\mathscr{H}_{R, C_{R}}$, on the set $R$ consisting of all restrictions of $\mathscr{H}$ to the set $R$ [1]. We let $\|\cdot\|_{C}$ and $\|\cdot\|_{R, C_{R}}$ denote the norms on $\mathscr{H}$ and $\mathscr{H}_{R, C_{R}}$, respectively. Then $\|\cdot\|_{R, C_{R}}$ is given by for each $f \in \mathscr{H}_{R, C_{R}}$,

$$
\|f\|_{R, C_{R}}:=\inf \left\{\|\tilde{f}\|_{C}: \tilde{f}(x)=f(x) \text { for all } x \in R\right\} .
$$

Moreover, for each $f \in \mathscr{H}_{R, C_{R}}$, there is a unique vector $f^{\prime} \in \mathcal{H}$ whose restriction to $R$ is $f$ and

$$
\|f\|_{R, C_{R}}=\left\|f^{\prime}\right\|_{C} \text {. }
$$

By the restriction theory for the RKHS's, the key terms $\alpha(x ; \xi)-\alpha(x ; u \xi)$ in the above proposition have the following representations (see [27, Proposition 3.2]):

Proposition 3.8. For any $x \neq u \in E$ and $x, u \notin \xi \subset E$, we have

$$
\alpha(x ; \xi)-\alpha(x ; u \xi)=\left|\left(e_{x}, e_{u}\right)_{\xi^{c}, B_{\xi^{c}}}\right|^{2}\left\|e_{u}\right\|_{\xi^{c}, B_{\xi^{c}}}^{-2} .
$$

In particular, in a formal way, we also have the representation:

$$
\alpha(x ; \xi)-\alpha(x ; u \xi)=\left|A(x, u)-A(x, \xi) A(\xi, \xi)^{-1} A(\xi, u)\right|^{2} \alpha(u ; \xi)^{-1}
$$

In the next section we will discuss some sufficient conditions so that we can control $\alpha(x ; \xi)-\alpha(x ; u \xi)$ uniformly for $\xi$.

\section{Examples}

In this section we discuss some examples for which the resulting Glauber and Kawasaki dynamics are Fellerian Markov processes on $C(X)$ and leave invariant certain DPP's. In Proposition 3.7 we have seen that if we could control the interdependencies; $M_{1}^{(\#)}<\infty$, then we are done. Unfortunately we couldn't do it under our hypothesis (H), so we impose further stronger conditions on the operator $A$. For any complex number $z \in \mathbb{C}$, we let $|z|_{1}:=|\operatorname{Re} z|+|\operatorname{Im} z|$.

Assumption (A): Let $A$ be a positive definite, bounded linear operator on $\mathcal{H}_{0} \equiv l^{2}(E)$. We assume that there is a $\lambda>0$ such that

$$
\inf _{x \in E}\left(A(x, x)-\sum_{y \neq x}|A(x, y)|_{1}\right) \geq \lambda .
$$

Any operator $A$ that satisfies the Assumption (A) is said to be diagonally dominant. When one considers the convolution operators on $l^{2}\left(\mathbb{Z}^{d}\right)$, it is not hard to see that there are many operators $A$ that satisfy the assumption (A). For example, let $C(x, y) \equiv C(x-y)$ be the convolution operator on $l^{2}\left(\mathbb{Z}^{d}\right)$ coming from the Fourier coefficients $C(\cdot)$ of a sufficiently smooth positive function $\rho(t)$ on the torus $\mathbb{T}^{d}$. Then the components $C(x, y)$ decrease fast enough so that $\sum_{y}|C(x, y)|<\infty$. We may then take $A:=a I+C$ for some positive numbers $a$.

Throughout this section we suppose that our operators $A$ satisfy the assumption (A). Let us fix a constant $q>0$ so that

$$
q \geq q(A):=\sup _{x \in E} \sum_{y \neq x}|A(x, y)|_{1}
$$

We define a $Q$-matrix $\widehat{Q}$ on $E$ by

$$
\widehat{Q}(x, y)= \begin{cases}\frac{1}{q}|A(x, y)|_{1}, & x \neq y \\ -\sum_{y \neq x} \frac{1}{q}|A(x, y)|_{1}, & x=y .\end{cases}
$$

Let $\widehat{\Pi}$ be a stochastic matrix on $E$ defined by

$$
\widehat{\Pi}:=\widehat{Q}+I \text {. }
$$

The following lemma is an analogue of [22, Lemma 4.1]. The proof follows by modifying the idea of the proof in [22] and we leave it at the appendix for readers' convenience.

Lemma 4.1. Assume that the operator A satisfies the assumption (A). Then for any $\xi \subset E$, the submatrix $A(\xi, \xi)$ is invertible and for any $x, y \in \xi$, 


$$
\left|A(\xi, \xi)^{-1}(x, y)\right| \leq M(x, y):= \begin{cases}\frac{1}{\lambda}, & x=y \\ \frac{1}{\lambda} \Gamma(x, y), & x \neq y .\end{cases}
$$

where $\Gamma:=\sum_{n=1}^{\infty}\left(\frac{q}{\lambda+q} \widehat{\Pi}\right)^{n}$.

With the help of the above lemma, we are able to state our main result.

Theorem 4.2. Suppose that the operator A satisfies the assumption (A). Let us take the flip rates $b(x ; \xi), d(x ; \xi)$, and $c(x, y ; \xi)$ as stated in Proposition 3.7. Then the hypotheses of Theorem 3.1 are satisfied and hence all the statements of Theorem 3.1 hold and the DPP $\mu$ corresponding to the kernel operator $K:=A(I+A)^{-1}$ is invariant under the Glauber and Kawasaki dynamics. Further, in addition to it, if $q(A)$ in (4.1) is sufficiently small, then the Markov processes are ergodic and the statements in Theorem 3.2 hold.

Proof. For the existence of Markov processes for Glauber and Kawasaki dynamics, by Proposition 3.7, it is enough to check that $M_{1}^{(\#)}$, \#=G or K, defined in (3.16)-(3.17), are finite. Under the assumption (A), (3.21) has a rigorous meaning. By Lemma 4.1, $A$ and any submatrix of $A$ are boundedly invertible and we have $\left\|A(\xi, \xi)^{-1}\right\| \leq(\lambda+q) / \lambda^{2}$, uniformly for $\xi \in E$. Then we easily see that $\alpha(x ; \xi)=\left(A(x \xi, x \xi)^{-1}(x, x)\right)^{-1}$, and Lemma 4.1 gives the bound:

$$
\alpha(x ; \xi) \geq \lambda, \quad \text { uniformly for } x \in E \text { and } x \notin \xi \subset E .
$$

By using again the result of Lemma 4.1 in (3.21) we see that

$$
\begin{aligned}
\sup _{\xi \ngtr x, u}\left|A(x, \xi) A(\xi, \xi)^{-1} A(\xi, u)\right| & \leq \sup _{\xi \ngtr x, u} \sum_{y, z \in \xi}|A(x, y)| M(y, z)|A(z, u)| \\
& \leq \sum_{y, z \in E \backslash\{x, u\}}|A(x, y)| M(y, z)|A(z, u)| .
\end{aligned}
$$

Hence we have by (3.21) and (4.5)-(4.6)

$$
\begin{aligned}
M_{1}^{(\mathrm{G})} & =\sup _{x \in E} \sum_{u \neq x} \sup _{\xi \ngtr x, u}(\alpha(x ; \xi)-\alpha(x ; u \xi)) \\
& \leq \sup _{x \in E} \sum_{u \neq x}\left(|A(x, u)|+\sum_{y, z \in E \backslash\{x, u\}}|A(x, y)| M(y, z)|A(z, u)|\right) \frac{1}{\lambda} \\
& \leq\left(q+q^{2} \frac{\lambda+q}{\lambda^{2}}\right) \frac{1}{\lambda}=\frac{q}{\lambda}\left(1+q \frac{\lambda+q}{\lambda^{2}}\right)<\infty .
\end{aligned}
$$

In a very similar way it follows that $M_{1}^{(\mathrm{K})}<\infty$.

The flip or jump rates are chosen so that the Markov processes satisfy the detailed balance conditions w.r.t. the DPP $\mu$. So, $\mu$ is reversible for both Glauber and Kawasaki dynamics, and hence $\mu$ is invariant under the dynamics (see [9, Propositions 5.2 and 5.3, Chapter II]).

In order to check the ergodicity we have to know the quantities $\varepsilon^{(\#)}$, \# = G or K. By using the definition of the flip and jump rates and also by using the property (4.5) it is not hard to check that

$$
\varepsilon^{(\mathrm{G})}=1 \quad \text { and } \quad \varepsilon^{(\mathrm{K})} \geq d_{1} \lambda \frac{1}{(1+\|A\|)^{2 t}},
$$

where $d_{1}$ is the constant in (3.15) and $\|A\|$ is the operator norm of $A$. By Proposition 3.7, (4.7), and (4.8), we see that if $q$ is small enough, then $M^{(\#)}<\varepsilon^{(\#)}$, and this completes the proof.

\section{Appendix}

In this appendix we provide with the proof of Lemma 4.1. The main ingredients of the method are the Markov chain on the discrete set E, Feynman-Kac formula, and the comparison of stochastic matrices of the Markov chains. The central idea was introduced in the proof of [22, Lemma 4.1].

Proof of Lemma 4.1. We first assume that $A$ is a real matrix. For each $\xi \subset E$, we define a $Q$-matrix $Q_{\xi}$ on the set $\xi \cup \bar{\xi} \cup\{\partial\}$, where $\partial$ is an abstract extra point playing as a cemetery (when $\xi \equiv E$, we just ignore it), and $\bar{\xi}$ is also an abstract set consisting of the elements of copies of $\xi$; we denote them as $\bar{\xi}:=\{\bar{x}: x \in \xi\}$. We define

$$
\begin{aligned}
& Q_{\xi}(x, y) \equiv Q_{\xi}(\bar{x}, \bar{y}):=\frac{1}{q} A(x, y)_{-}, \quad x \neq y \in \xi, \\
& Q_{\xi}(x, \bar{y}) \equiv Q_{\xi}(\bar{x}, y):=\frac{1}{q} A(x, y)_{+}, \quad x \neq y \in \xi,
\end{aligned}
$$




$$
\begin{aligned}
& Q_{\xi}(x, \bar{x}) \equiv Q_{\xi}(\bar{x}, x):=0, \quad x \in \xi, \\
& Q_{\xi}(x, x) \equiv Q_{\xi}(\bar{x}, \bar{x}):=-\sum_{y \in E ; y \neq x} \frac{1}{q}|A(x, y)|, \quad x \in \xi, \\
& Q_{\xi}(x, \partial) \equiv Q_{\xi}(\bar{x}, \partial):=\sum_{y \in E \backslash \xi} \frac{1}{q}|A(x, y)|, \quad x \in \xi, \\
& Q_{\xi}(\partial, x) \equiv Q_{\xi}(\partial, \bar{x}) \equiv Q_{\xi}(\partial, \partial):=0, \quad x \in \xi,
\end{aligned}
$$

here $a_{ \pm}:=\max \{ \pm a, 0\}$ for each real number $a$. Given a function $f \in l^{\infty}(\xi)$, we extend it to a function $\tilde{f} \in$ $l^{\infty}(\xi \cup \bar{\xi} \cup\{\partial\})$ by

$$
\tilde{f}(\tilde{x}):= \begin{cases}f(x), & \tilde{x}=x \\ -f(x), & \tilde{x}=\bar{x} \\ 0, & \tilde{x}=\partial .\end{cases}
$$

We consider the anti-symmetric subspace $l_{a}^{\infty}(\xi \cup \bar{\xi} \cup\{\partial\})$ of $l^{\infty}(\xi \cup \bar{\xi} \cup\{\partial\})$ defined by

$$
l_{a}^{\infty}(\xi \cup \bar{\xi} \cup\{\partial\}):=\left\{f \in l^{\infty}(\xi \cup \bar{\xi} \cup\{\partial\}): f(\bar{x})=-f(x), f(\partial)=0\right\} .
$$

We notice that $Q_{\xi}$ can be regarded as an operator on $l_{a}^{\infty}(\xi \cup \bar{\xi} \cup\{\partial\})$. We define a function $V_{\xi}(\widetilde{x})$ on $\xi \cup \bar{\xi} \cup\{\partial\}$ by for $x \in \xi$,

$$
V_{\xi}(x)=V_{\xi}(\bar{x}):=\frac{1}{q}\left(-A(x, x)+\sum_{y \in E: y \neq x}|A(x, y)|\right), \quad \text { and } \quad V_{\xi}(\partial)=0 .
$$

Now we consider the equation:

$$
(A(\xi, \xi) f)(x)=h(x), \quad x \in \xi,
$$

for $h \in l^{\infty}(\xi)$. By the definition of the $Q$-matrix $Q_{\xi}$, the equation (A.5) is equivalent to

$$
-\left(Q_{\xi}+V_{\xi}\right) \tilde{f}=\frac{1}{q} \tilde{h}
$$

Let us define a stochastic matrix $\Pi_{\xi}$ on $\xi \cup \bar{\xi} \cup\{\partial\}$ by

$$
\Pi_{\xi}:=Q_{\xi}+I_{\xi \cup \bar{\xi} \cup\{\partial\}} .
$$

Let $\left\{X_{t}^{(\xi)}: t \geq 0\right\}$ be the Markov chain on $\xi \cup \xi \cup\{\partial\}$ generated by $Q_{\xi}$. Then by the Feynman-Kac formula, we have

$$
\begin{aligned}
\tilde{f}(\widetilde{x}) & =-\frac{1}{q}\left(Q_{\xi}+V_{\xi}\right)^{-1} \tilde{h}(\widetilde{x}) \\
& =\frac{1}{q} \mathbb{E}_{\tilde{x}}\left[\int_{0}^{\infty} \tilde{h}\left(X_{t}^{(\xi)}\right) \exp \left(\int_{0}^{t} V_{\xi}\left(X_{s}^{(\xi)}\right) d s\right) d t\right] .
\end{aligned}
$$

Let us now take $h:=\delta_{y}$, the Dirac function at the point $y$. Then the solution $f(x)$ in $(\mathrm{A} \cdot 5)$ is $f(x)=A(\xi, \xi)^{-1}(x, y)$, thus we have (notice that $\tilde{\delta}_{y}=\delta_{y}-\delta_{\bar{y}}$ )

$$
\left|A(\xi, \xi)^{-1}(x, y)\right| \leq \frac{1}{q} \mathbb{E}_{x}\left[\int_{0}^{\infty} \delta_{y}\left(\pi\left(X_{t}^{(\xi)}\right)\right) \exp \left(\int_{0}^{t} V_{\xi}\left(\pi\left(X_{s}^{(\xi)}\right)\right) d s\right) d t\right],
$$

where $\pi$ is a projection operator on $\xi \cup \bar{\xi} \cup\{\partial\}$ defined by

$$
\pi(x) \equiv \pi(\bar{x}):=x \quad \text { and } \quad \pi(\partial):=\partial .
$$

We would like to estimate the r.h.s. of (A.9). For this, we introduce another $Q$-matrix $\widehat{Q}_{\xi}$ on $\xi \cup\{\partial\}$ by

$$
\begin{aligned}
& \widehat{Q}_{\xi}(x, y)= \begin{cases}\frac{1}{q}|A(x, y)|, & x \neq y, x, y \in \xi, \\
-\frac{1}{q} \sum_{z \in E: z \neq x}|A(x, z)|, & x=y \in \xi,\end{cases} \\
& \widehat{Q}_{\xi}(x, \partial)=\frac{1}{q} \sum_{z \in E \backslash \xi}|A(x, z)|, \quad x \in \xi, \\
& \widehat{Q}_{\xi}(\partial, y) \equiv \widehat{Q}_{\xi}(\partial, \partial)=0, \quad y \in \xi .
\end{aligned}
$$

We also define a stochastic matrix $\widehat{\Pi}_{\xi}$ on $\xi \cup\{\partial\}$ by

$$
\widehat{\Pi}_{\xi}:=\widehat{Q}_{\xi}+I_{\xi \cup\{\partial\}} .
$$


Then we notice that the probability law of the chain $\left\{\pi\left(X_{t}^{(\xi)}\right)\right\}$ on $\xi \cup\{\partial\}$ is the same as that of the Markov chain on $\xi \cup\{\partial\}$ generated by $\widehat{Q}_{\xi}$. By the assumption (A) we notice that $V_{\xi} \leq-\lambda / q$. Thus by using the strong Markov property we get

$$
\left|A(\xi, \xi)^{-1}(x, y)\right| \leq \frac{1}{q}\left\|\left(\widehat{Q}_{\xi}+V_{\xi}\right)^{-1}\right\|_{\infty} \mathbb{E}_{x}\left[e^{-\lambda / q \tau_{y}^{(\xi)}} ; \tau_{y}^{(\xi)}<\infty\right]
$$

where $\tau_{y}^{(\xi)}$ is the first hitting time at $y$ of the chain $\left\{\pi\left(X_{t}^{(\xi)}\right)\right\}$, and $\left\|\left(\widehat{Q}_{\xi}+V_{\xi}\right)^{-1}\right\|_{\infty}$ is the operator norm of $\left(\widehat{Q}_{\xi}+V_{\xi}\right)^{-1}$ acting on $l^{\infty}(\xi \cup\{\partial\})$, which satisfies the bound:

$$
\left\|\left(\widehat{Q}_{\xi}+V_{\xi}\right)^{-1}\right\|_{\infty} \leq q / \lambda
$$

Since $\tau_{x}^{(\xi)}=0$ for the Markov process starting at $x$, we see from (A.13) and (A.14) that

$$
\left|A(\xi, \xi)^{-1}(x, x)\right| \leq 1 / \lambda \text {. }
$$

This is the bound in the lemma for the diagonal components. In order to estimate $\left|A(\xi, \xi)^{-1}(x, y)\right|$ for $x \neq y$, we define

$$
u_{y}^{(\xi)}(x):=\mathbb{E}_{x}\left[e^{-\lambda / q \tau_{y}^{(\xi)}} ; \tau_{y}^{(\xi)}<\infty\right], \quad x \neq y
$$

If we let $\tau$ the random variable which is exponentially distributed with parameter 1 , then we have the identity:

$$
\begin{aligned}
u_{y}^{(\xi)}(x) & =\mathbb{E}\left[e^{-\lambda / q \tau}\right]\left[\widehat{\Pi}_{\xi}(x, y)+\sum_{z \in \xi: z \neq y} \widehat{\Pi}_{\xi}(x, z) u_{y}^{(\xi)}(z)\right] \\
& =\left(\frac{q}{\lambda+q}\right)\left[\widehat{\Pi}_{\xi}(x, y)+\sum_{z \in \xi: z \neq y} \widehat{\Pi}_{\xi}(x, z) u_{y}^{(\xi)}(z)\right] \\
& =\sum_{n=1}^{\infty}\left(\frac{q}{\lambda+q} \widehat{\Pi}_{\xi}\right)^{n}(x, y) .
\end{aligned}
$$

Now by the definition of $\widehat{Q}_{\xi}(x, y)$ in $(\mathrm{A} \cdot 11)$ we see that for $\xi \subset \xi^{\prime}$,

$$
\widehat{\Pi}_{\xi}(x, y)=\widehat{\Pi}_{\xi^{\prime}}(x, y) \quad \text { whenever } x, y \in \xi \text {. }
$$

Also, since $\partial$ plays as a cemetery, once the process visits $\partial$, it never comes out from it. Therefore, the nonzero contributions in the term $\left(\widehat{\Pi}_{\xi}\right)^{n}(x, y)$ come only from the random walk path of length $n$ connecting $x$ and $y$ on the set $\xi$ (avoiding the cemetery d). Obviously, such a number of paths increases as the set $\xi$ increases. Therefore the last presentation of $(\mathrm{A} \cdot 17)$ is bounded by $\Gamma(x, y)$ where

$$
\Gamma:=\sum_{n=1}^{\infty}\left(\frac{q}{\lambda+q} \widehat{\Pi}\right)^{n},
$$

with $\widehat{\Pi}:=\widehat{\Pi}_{E}$. Inserting this and (A-14) into (A-13) we get the bound in the lemma for off-diagonal components. Together with (A.15) we are done in the case that $A$ is a real matrix. When $A$ is a complex matrix, we write $A=A_{1}+i A_{2}$, where $A_{1}$ and $A_{2}$ are real matrices. Let $E_{1}$ and $E_{2}$ be two copies of $E$. Then we have the bijection

$$
l^{2}(E) \ni f=f_{1}+i f_{2} \mapsto f_{1} \oplus f_{2} \in l_{\text {real }}^{2}\left(E_{1}\right) \oplus l_{\text {real }}^{2}\left(E_{2}\right) \cong l_{\text {real }}^{2}\left(E_{1} \cup E_{2}\right),
$$

where $l_{\text {real }}^{2}(\cdot)$ means the real Hilbert space. Under this map, $A$ in $l^{2}(E)$ is equivalent to the matrix

$$
\widetilde{A}=\left(\begin{array}{cc}
A_{1} & -A_{2} \\
A_{2} & A_{1}
\end{array}\right)
$$

acting on $l_{\text {real }}^{2}\left(E_{1} \cup E_{2}\right)$. Similary, for any subset $\xi \subset E$, the submatrix $A(\xi, \xi)$ acting on $l^{2}(\xi)$ is equivalent to the submatrix

$$
\widetilde{A}(\xi, \xi)=\left(\begin{array}{cc}
A_{1}(\xi, \xi) & -A_{2}(\xi, \xi) \\
A_{2}(\xi, \xi) & A_{1}(\xi, \xi)
\end{array}\right)
$$

of $\widetilde{A}$ acting on $l_{\text {real }}^{2}\left(\xi_{1} \cup \xi_{2}\right)$, where $\xi_{i}, i=1,2$, are again the copies of $\xi$. Notice that the enlarged real matrix $\widetilde{A}$ satisfies the conditions of the lemma with $E$ being replaced by $E_{1} \cup E_{2}$. Let $A(\xi, \xi)^{-1} \equiv C+i D$, where $C$ and $D$ are real matrices. Then we can check that

$$
C=P_{\xi_{1}} \widetilde{A}(\xi, \xi)^{-1} P_{\xi_{1}} \text { and } \quad D=-P_{\xi_{1}} \widetilde{A}(\xi, \xi)^{-1} P_{\xi_{2}},
$$

where $P_{\xi_{i}}, i=1,2$, are the projections on $l_{\text {real }}^{2}\left(\xi_{1} \cup \xi_{2}\right)$ onto $l_{\text {real }}^{2}\left(\xi_{i}\right), i=1,2$, respectively. So, $\left|A(\xi, \xi)^{-1}(x, y)\right| \leq$ $|C(x, y)|+|D(x, y)|$, and by $(\mathrm{A} \cdot 23)$ and applying the result for the real case we also arrive at the conclusion for the complex case. 


\section{Acknowledgments}

We thank the referee for valuable comments which improved the paper greatly. This work was supported by the Korea-Japan Basic Scientific Cooperation Program "Noncommutative Stochastic Analysis and Its Applications to Network Science."

\section{REFERENCES}

[1] Aronszajn, N., “Theory of reproducing kernels," Trans. Amer. Math. Soc., 68: 337-404 (1950).

[2] Georgii, H.-O., Gibbs measures and phase transitions, Walter de Gruyter, Berlin, New York (1988).

[3] Glötzl, E., "Time reversible and Gibbsian point process I. Markovian spatial birth and death processes on a general phase space," Math. Nachr., 102: 217-222 (1981).

[4] Glötzl, E., "Time reversible and Gibbsian point process II. Markovian particle jump processes on a general phase space," Math. Nachr., 106: 63-71 (1982).

[5] Holley, R. A., and Stroock, D. W., "Nearest neighbor birth and death processes on the real line," Acta Math., 140: 103-154 (1987).

[6] Katori, M., and Tanemura, H., "Noncolliding Brownian motion and determinantal processes,” J. Stat. Phys., 129: 1233-1277 (2007).

[7] Kondratiev, Y. G., and Lytvynov, E., "Glauber dynamics of continuous particle systems," Ann. Inst. H. Poincaré Probab. Statist., 41: 685-702 (2005)

[8] Kondratiev, Y. G., Lytvynov, E., and Röckner, M., "Equilibrium Kawasaki dynamics of continuous particle systems," Infin. Dimen. Anal. Quant. Prob. Rel. Top., 10(2): 185-209 (2007).

[9] Liggett, T. M., Interacting particle systems, Springer-Verlag, New York, Berlin, Heidelberg, Tokyo (1985).

[10] Lyons, R., "Determinantal probability measures," Publ. Math. Inst. Hautes Études Sci., 98: 167-212 (2003).

[11] Lytvynov, E., and Ohlerich, N., "A note on equilibrium Glauber and Kawasaki dynamics for fermion point processes," Methods Funct. Anal. Topology, 14(1): 67-80 (2008).

[12] Ma, Z. M., and Röckner, M., Introduction to the theory of (non-symmetric) Dirichlet forms, Springer-Verlag, Berlin, Heidelberg, New York (1992).

[13] Macchi, O., "The coincidence approach to stochastic point processes," Adv. Appl. Prob., 7: 83-122 (1975).

[14] Matthes, K., Warmuth, W., and Mecke, J., "Bemerkungen zu einer Arbeit von Nguyen Xuan Xanh und Hans Zessin," Math. Nachr., 88: 117-127 (1979).

[15] Nguyen, X. X., and Zessin, H., "Integral and differential characterizations of the Gibbs process," Math. Nachr., 88: 105-115 (1975).

[16] Osada, H., Interacting Brownian motions in infinite dimension with logarithmic interaction potentials, Preprint.

[17] Preston, C., "Spatial birth and death processes, in Proceedings of the 40th Session of the International Statistical Institute (Warsaw, 1975), Vol 2," Bull. Inst. Internt. Statist., 46: 371-391 (1975).

[18] Ruelle, D., "Superstable interaction in classical statistical mechanics," Comm. Math. Phys., 18: 127-159 (1970).

[19] Shiga, T., "Some problem related to Gibbs states, canonical Gibbs states and Markovian time evolutions," Z. Wahrsch. Verw. Gebiete, 39: 339-352 (1977).

[20] Shirai, T., and Takahashi, Y., "Random point field associated with certain Fredholm determinant I: fermion, Poisson, and boson point processes," J. Funct. Anal., 205: 414-463 (2003).

[21] Shirai, T., and Takahashi, Y., "Random point field associated with certain Fredholm determinant II: fermion shift and its ergodic and Gibbs properties," Ann. Prob., 31: 1533-1564 (2003).

[22] Shirai, T., and Yoo, H. J., "Glauber dynamics for fermion point processes," Nagoya Math. J., 168: 139-166 (2002).

[23] Soshnikov, A., "Determinantal random point fields," Russ. Math. Surv., 55: 923-975 (2000).

[24] Spohn, H., Interacting Brownian particles: a study of Dyson's model. In: Hydrodynamic behavior and interacting particle systems, Papanicolaou, G., ed., IMA Volumes in Mathematics and its Applications Vol 9, Springer-Verlag, Berlin, 151-179 (1987).

[25] Yoo, H. J., "Dirichlet forms and diffusionn processes for fermion random point fields," J. Funct. Anal., 219: 143-169 (2005).

[26] Yoo, H. J., "A variational principle in the dual pair of reproducing kernel Hilbert spaces and an application," J. Stat. Phys., 126(2): 325-354 (2007).

[27] Yoo, H. J., Dual spaces of restrictions in the reproducing kernel Hilbert spaces in discrete sets, to appear in Kodai Math. J. 